\title{
Direction-dependent excitatory and inhibitory ocular vestibular-evoked myogenic potentials (oVEMPs) produced by oppositely directed accelerations along the midsagittal axis of the head
}

\author{
Peter Jombik · Pavel Spodniak · Vladimír Bahyl
}

Received: 20 October 2010 / Accepted: 4 April 2011 / Published online: 22 April 2011

(C) The Author(s) 2011. This article is published with open access at Springerlink.com

\begin{abstract}
Oppositely directed displacements of the head need oppositely directed vestibulo-ocular reflexes (VOR), i.e. compensatory responses. Ocular vestibular-evoked myogenic potentials (oVEMPs) mainly reflect the synchronous extraocular muscle activity involved in the process of generating the VOR. The oVEMPs recorded beneath the eyes when looking up represent electro-myographic responses mainly of the inferior oblique muscle. We aimed: (1) to study the properties of these responses as they were produced by head acceleration impulses to the forehead and to the back of the head; (2) to investigate the relationships between these responses and the 3-D linear head accelerations that might reflect the true stimulus that acts on the vestibular hair cells. We produced backward- and forwarddirected acceleration stimuli in four conditions (positive and negative head acceleration impulses to the hairline and to the inion) in 16 normal subjects. The oVEMPs produced by backward- and forward-directed accelerations of the head showed consistent differences. They were opposite in the phase. The responses produced by backward accelera-
\end{abstract}

P. Jombik ( $₫)$

Department of Neurology, Laboratory of Clinical

Neurophysiology, Zvolen Hospital,

96001 Zvolen, Slovak Republic

e-mail: peter.jombik@post.sk

P. Spodniak

Department of Environmental Sciences,

Faculty of Environmental Technology,

Zvolen Technical University, Zvolen, Slovak Republic

V. Bahyl

Department of Physics, Electrotechnics and Applied Mechanics, Faculty of Wood Sciences, Zvolen Technical University,

Zvolen, Slovak Republic tions of the head began with an initial negativity, n11; conversely, those produced by accelerations directed forward showed initially a positive response, $\mathrm{p} 11$. There was a high inter-subject correlation of head accelerations along the head anteroposterior and transverse axes, but almost no correlation of accelerations along the vertical axis of the head. We concluded that backward-directed head accelerations produced an initial excitatory response, and forwarddirected accelerations of the head were accompanied by an initial inhibitory response. These responses showed dependence on acceleration direction in the horizontal plane of the head. This could be consistent with activation of the utricle.

Keywords Vestibulo-ocular reflex - Otolith - Utricle . Vestibular-evoked potentials $\cdot$ Head acceleration

\section{Introduction}

The vestibular system senses head displacements and, in accord with other sensory and motor systems, generates appropriate reflex responses to maintain balance of the body and position of the eyes in space (Baloh and Honrubia 2001). The main role of the vestibulo-ocular reflex (VOR) is to preserve clear vision especially during rapid head displacements (Angelaki and Dickman 2000; Highstein et al. 2005). This objective is achieved by the semicircular canals and otolithic organs and their projections to the ocular motor nuclei in the brainstem (Leigh and Zee 2006). The canals sense angular accelerations, the saccular afferents are predominantly activated by linear forces acting in the vertical plane, while the utricle responds mainly to linear accelerations in the horizontal plane (Goldberg and Fernandez 1971; Fernandez and Goldberg 1976; Raphan and Cohen 
2002). Several studies have recently investigated the electrical potentials that can be recorded close to the eyes in response to bone-conducted (BC) sound (Jombik and Bahyl 2005a; Iwasaki et al. 2007) and air-conducted (AC) sound (Halmagyi et al. 2003; Jombik and Bahyl 2005b; Aw et al. 2006; Chihara et al. 2007) as well as galvanic stimuli (Rosengren et al. 2009a, b). It is now generally accepted that these potentials mainly reflect the synchronous extraocular muscle activity that is involved in the process of generating the VOR (Rosengren et al. 2005; Todd et al. 2007). Nowadays, they are termed "ocular vestibularevoked myogenic potentials" (oVEMPs) (Todd et al. 2007). It is probable that many, if not all, eye muscles are activated by the above-mentioned stimuli. However, the most reproducible responses can be obtained by bipolar recording from sites beneath the eyes during upward gaze. In this instance, the inferior oblique muscle gets closest to the recording site, and thus the response reflects the activity of mainly this muscle. It has been shown that $\mathrm{AC}$ and $\mathrm{BC}$ oVEMPs can be valuable tools for clinical investigation of the vestibulo-ocular reflex circuits (Iwasaki et al. 2007; Rosengren et al. 2007, 2008; Welgampola 2008; Welgampola et al. 2008; Iwasaki et al. 2008b, 2009; Wang et al. 2010; Cheng et al. 2009; Manzari et al. 2010). However, the specificity of the primary vestibular afferents and their efferent projections that are activated by different kinds of stimuli, i.e. AC and BC sound, is still a matter of dispute. In our previous study using EOG montage, we showed a complementary mirror image of the responses with phase reversal of the recorded potentials that were produced by head taps to the mastoids, i.e. in response to acceleration along the head's transverse axis (Jombik and Bahyl 2005a). This finding was later confirmed by the oVEMP study of (Todd et al. 2008a). It is also in agreement with findings on the cervical-evoked myogenic potentials (cVEMPs) produced by lateral head taps (Brantberg and Tribukait 2002; Brantberg et al. 2009) and lateral impulse stimuli (Rosengren et al. 2009a, b). These findings might suggest utricular origin of the responses. Similar behaviour of the cVEMPs, and the periocular potentials produced by $\mathrm{BC}$ sound stimuli in the sagittal plane and recorded by EOG montage, has been shown in (Brantberg et al. 2008; Jombik et al. 2008). On the other hand, Iwasaki et al. (2008a) found no difference between oVEMPs produced by head taps to the hairline and inion. In another study (Lin et al. 2010), these responses differed in the latency of their first negative peak. The authors explained this finding by the differing conduction velocities of $\mathrm{BC}$ sound when stimuli were applied to the anterior sites of the head compared to the inion. The BC oVEMPs produced by stimuli applied to the midline of the head allow a simple and straightforward assessment of the symmetry of the vestibular function. Several studies have shown the reliability and efficacy of this method in a clinical setting (Iwasaki et al. 2008b, 2009; Wang et al. 2010; Manzari et al. 2010).

In the current study, we attempt to investigate the differences between the oVEMPs produced by BC stimuli to the hairline versus the inion and the relationship of these responses to head accelerations recorded close to the otic capsule at the mastoid. We also want to clarify some of the above-mentioned controversies regarding the BC oVEMP produced at midsagittal cranial sites.

\section{Methods}

Subjects

Sixteen healthy volunteers (mean age 38.3 years; SD 13.5) participated in the study. That included eight women (mean age 36.1 years; SD 12.1) and eight men (mean age 41.5 years; SD 14.8). All participants gave informed consent in line with the Helsinki Declaration, and the study was approved by the local ethics committee.

\section{Experimental procedure}

The experiment consisted of two parts. In the first part, EMG responses of the oVEMPs were produced and recorded. The other part consisted of the recording of head accelerations.

Stimulation

The stimuli consisted of positive and negative impulses. The driving electrical waveforms for the impulses (positive or negative square waves with duration of $1 \mathrm{~ms}$ ) were delivered by the sound generator of a 5-channel EMG/EP system (Teca Synergy, Medelec, UK), and the same apparatus was used for recording the EMG signals and the head accelerations. The impulses were amplified and low-pass filtered at $250 \mathrm{~Hz}$ by an external amplifier and applied using a custom-made mini-shaker at a rate of 3 per second and 1 per second for producing oVEMP and recording head accelerations, respectively. The mini-shaker was fitted with a short $2-\mathrm{cm}$ bolt terminating in a bakelite cap $1.5 \mathrm{~cm}$ in diameter, which was the contact point for the stimulator on the subject's head. The stimulator was held normal to the subject's head with an approximate force of $10-20 \mathrm{~N}$ by the experimenter, who stood behind or in front of the subject according to the site of stimulation. Owing to the electromechanical properties of the mini-shaker, the filtering and the stimulus application mode, the main frequency of the resultant output impulse was about $100 \mathrm{~Hz}$. The resultant output represented the impulse response of the coupled shaker-head system. The net effect was the acceleration of 
this coupled shaker-head system that was directed alternately away from and towards the experimenter by the positive and the negative impulses, respectively.

\section{OVEMP recordings}

EMG signals were recorded by bipolar montage. The active (inverting) electrode was placed on the orbital margin below the eye and referred to an electrode approximately $20 \mathrm{~mm}$ below it on the cheek, and the ground electrode was placed on the sternum. The EMG signals were amplified and band-pass filtered $(5-500 \mathrm{~Hz})$, and sampled at $20 \mathrm{kHz}$ for $50 \mathrm{~ms}$ following stimulus onset. During stimulation, the subjects were seated comfortably and instructed to look up and relax their jaw muscles. EMG responses to 30 stimuli were averaged for each of the four conditions, i.e. positive and negative impulses that were subsequently applied on the subject's head at $\mathrm{Fz}^{1}$ (midline forehead at hairline) and inion.

\section{Accelerometry}

In the second part of the experiment, linear acceleration of the head was recorded using a custom-made miniature triaxial accelerometer consisting of two ADXL311 dual-axis analogue devices cemented together and embedded in synthetic resin. Another dual-axis accelerometer of the same type was fitted to the bottom of a cylindrical plastic nozzle that now covered the bakelite cap of the stimulator. Thus, the active axis of the accelerometer, which was oriented normal to the head, directly recorded the acceleration impulse at the contact sites of the stimulator and the head, because the acceleration pulse was transmitted through the accelerometer. Linear acceleration of the head was produced by the same stimuli used for producing EMG responses and was recorded at the left mastoid bone site approximately $1 \mathrm{~cm}$ behind the pinna of the ear at the level of the tragus. To ensure tight contact of the accelerometer with the head surface, the subjects pressed the miniature device to their heads with their index fingers. The placement of the accelerometer was constant in all recordings, and its axes were oriented in parallel with the anteroposterior $X$, the vertical $Z$ and the transversal $Y$ axes of the head. The 3-D accelerometer signal was amplified and band-pass filtered $(0.2-500 \mathrm{~Hz})$, and sampled at $20 \mathrm{kHz}$ for $50 \mathrm{~ms}$ following stimulus onset. Five to ten head acceleration stimuli were averaged for each condition.

\footnotetext{
${ }^{1}$ International $10-20$ system for electroencephalographic electrode placements, according to recommendation of the Committee of the International Federation of Societies for Electroencephalography and Clinical Neurophysiology (Jasper 1958).
}

Auxiliary experiments

The applied impulses could cause not only translational but also rotational displacement of the head; thus, they could stimulate not only the afferents of the otolithic organs but also the canal afferents. In order to pursue this possibility, the experimental procedure was repeated in a fixed head condition in one subject. The subject (one of the authors, PJ) pressed his head to the wall on the side opposite to the site of stimulation.

Data analysis

Latencies and amplitudes were measured for both the EMG and accelerometer signals off line. The latencies were analysed both for their absolute values and for their values corrected to the mini-shaker acceleration onsets. Absolute amplitudes were measured at the response peaks, and relative amplitudes were calculated as the differences between the subsequent oppositely directed peaks. Repeated measures ANOVAs and $t$ tests when appropriate were used to compare response amplitudes and latencies. Reproducibility was assessed with Pearson's correlations. Frequency analysis of the stimulus and accelerometry records at the mastoid and of the EMG responses was performed on grand averages of the traces using FFT. Values reported are means $\pm \mathrm{SD}$. For conciseness, only the results of the acceleration onsets and the first acceleration peaks recorded at the mini-shaker and at the mastoid site and the initial two peaks of the VEMPs are displayed here.

\section{Results}

Accelerometry measurements on the mini-shaker

Owing to the electro-mechanical delay of the hardware, the onset of mini-shaker acceleration began at about $0.50 \mathrm{~ms}$ after the trigger. It reached its peak value of about $1.3 \mathrm{~g}$ at $2.20 \mathrm{~ms}$ (Table 1). There were no statistically significant differences in the acceleration latency and magnitude at this first peak between the four experimental conditions. However, there was a small but statistically significant difference in the mini-shaker acceleration onset between stimuli applied to the hairline at Fz and to the inion $(P<0.01)$. Latency was about $30 \mu$ s shorter in stimuli applied to the inion compared to stimulation at Fz. On the other hand, there were no significant differences between the latencies of the first peak of mini-shaker acceleration.

Accelerometry measurements at the mastoid. Comparison of the findings on individual ( $x, y$ and $z$ ) axes between the four experimental conditions

Onset of acceleration along all 3 axes of the accelerometer at the mastoid was between 1.5 and $2.5 \mathrm{~ms}$, while the peak 
Table 1 Accelerometry findings at the interface of the mini-shaker and the head

\begin{tabular}{|c|c|c|c|c|}
\hline & \multicolumn{2}{|c|}{ Acceleration backward } & \multicolumn{2}{|c|}{ Acceleration forward } \\
\hline & Fz plus & Inion minus & Inion plus & Fz minus \\
\hline \multicolumn{5}{|c|}{ Ons lat (ms) } \\
\hline Mean & 0.51 & $0.48 * *$ & $0.48 * *$ & 0.51 \\
\hline SD & 0.06 & 0.06 & 0.04 & 0.05 \\
\hline \multicolumn{5}{|c|}{ Peak 1 lat (ms) } \\
\hline Mean & 2.16 & 2.21 & 2.18 & 2.18 \\
\hline SD & 0.05 & 0.06 & 0.04 & 0.07 \\
\hline \multicolumn{5}{|c|}{ Ons-peak 1 amp (g) } \\
\hline Mean & 1.22 & 1.31 & 1.27 & 1.23 \\
\hline SD & 0.20 & 0.14 & 0.14 & 0.19 \\
\hline
\end{tabular}

Ons onset, Lat latency, $g=9.81 \mathrm{~m} \mathrm{~s}^{-2}$

** Statistical significance level at $P<0.01$ for shorter latencies of the acceleration onsets in stimuli applied to the inion

values of about $0.02-0.06 \mathrm{~g}$ were reached between 3.5 and $5.5 \mathrm{~ms}$ (Table 2). There were no significant differences either between the latencies of acceleration onsets or the latencies of first peaks along the head $X$ axis. On the other hand, the latencies of the onsets and the first peaks along the $Y$ axis as well as the latencies of the first peaks along the $Z$ axis were significantly shorter for stimuli applied to $\mathrm{Fz}$ $(P<0.05)$. The magnitudes of the first acceleration peaks along all the axes in the four experimental conditions did not show significant differences.

However, the orientations of the acceleration vectors along the head $X$ and $Y$ axes showed consistent differences that were dependent on the initial direction of the stimulus delivered by the mini-shaker, i.e. the direction of minishaker acceleration. Positive impulses applied to $\mathrm{Fz}$ and negative impulses at the inion caused backward-directed head acceleration along the head $\mathrm{X}$ axis and outward (laterally)-directed acceleration along the head $Y$ axis (Fig. 1). In contrast, positive impulses applied to the inion and negative impulses applied to Fz resulted in forward-directed acceleration along the head $X$ axis and inward (medially)-directed acceleration along the $Y$ axis (Fig. 2). Thus, the acceleration vectors along the head $X$ and $Y$ axes were out of phase with forward- and backward-directed stimuli.

The acceleration directions along the head $\mathrm{X}$ axis were identical in all test subjects, and the mean values of interindividual Pearsons's correlation coefficients " $r$ " of the acceleration traces during the first $6 \mathrm{~ms}$ showed very high correlations $(+\mathrm{Fz} 0.84,+$ Inion $0.93,-$ Inion 0.90 , and $-\mathrm{Fz}$ $0.80)$.

In one subject, the acceleration vectors along the head $Y$ axis were inverse compared to the rest of the test subjects for stimulation at Fz. Another two subjects were inverse to the rest of the subjects for stimuli applied to the inion. All these three "inverse" subjects were men. Despite these few exceptions, the mean values of the inter-subject correlation

Table 2 Accelerometry findings at the mastoid site

\begin{tabular}{|c|c|c|c|c|c|c|c|c|c|c|c|c|}
\hline & \multicolumn{6}{|c|}{ Acceleration backward } & \multicolumn{6}{|c|}{ Acceleration forward } \\
\hline & \multicolumn{3}{|l|}{ Fz plus } & \multicolumn{3}{|c|}{ Inion minus } & \multicolumn{3}{|c|}{ Inion plus } & \multicolumn{3}{|l|}{ Fz minus } \\
\hline & $X$ & $Y$ & $Z$ & $X$ & $Y$ & $Z$ & $X$ & $Y$ & $Z$ & $X$ & $Y$ & $Z$ \\
\hline \multicolumn{13}{|c|}{ Ons (ms) } \\
\hline Mean & $2.09 * *$ & $1.53^{\#}$ & $2.18^{* *}$ & $2.14 * *$ & 1.69 & 2.09 & $2.31 * *$ & 1.79 & $2.32 *$ & $1.98 *$ & $1.53^{\#}$ & $2.09 * *$ \\
\hline SD & 0.54 & 0.41 & 0.43 & 0.39 & 0.26 & 0.78 & 0.30 & 0.38 & 0.67 & 0.54 & 0.39 & 0.43 \\
\hline \multicolumn{13}{|c|}{ Peak 1 lat (ms) } \\
\hline Mean & $4.99 * *^{\dagger}$ & $3.98^{\#}$ & $4.44 * \# \#$ & $4.86^{* *}$ & 4.11 & $4.89^{* *}$ & $5.08 * *$ & 4.26 & $4.96 * *$ & $5.06 * * \dagger \dagger$ & $3.89^{\#}$ & $4.41 * * \# \#$ \\
\hline SD & 0.46 & 0.26 & 0.73 & 0.56 & 0.56 & 0.61 & 0.35 & 0.48 & 0.74 & 0.57 & 0.32 & 0.64 \\
\hline \multicolumn{13}{|c|}{ Ons-peak 1 amp (g) } \\
\hline Mean & $0.06 * *$ & 0.05 & 0.03 & $0.05 * *$ & 0.04 & 0.03 & $0.06^{* *}$ & $0.04 *$ & 0.03 & $0.05 * *$ & 0.04 & 0.03 \\
\hline SD & 0.03 & 0.03 & 0.02 & 0.02 & 0.02 & 0.01 & 0.02 & 0.02 & 0.01 & 0.03 & 0.03 & 0.02 \\
\hline
\end{tabular}

Ons onset, Lat latency, Sh mini-shaker, $g=9.81 \mathrm{~m} \mathrm{~s}^{-2}$

\# and ${ }^{\# \#}$ statistical significance level at $P<0.05$ and $P<0.01$ for shorter latencies of the acceleration onsets and the first peaks on the $Y$ and $Z$ axes for stimuli applied to $\mathrm{Fz}$ in the between-conditions comparisons

$*$ and ** statistical significance level at $P<0.05$ and $P<0.01$, respectively, for longer latencies of the acceleration onsets and the first peaks on the $X$ and $Z$ axes compared to the $Y$ axis in the within-conditions comparisons

$\dagger$ and ${ }^{\dagger \dagger}$ statistical significance level at $P<0.05$ and $P<0.01$, respectively, for longer latencies of the acceleration first peaks on the $X$ axis compared to the $Z$ axis in the within-conditions comparisons

* and ** statistical significance level at $P<0.05$ and $P<0.01$, respectively, for higher amplitudes of the first acceleration peaks on the $X$ and the

$Y$ axes compared to the $Z$ axis in the within-conditions comparisons 

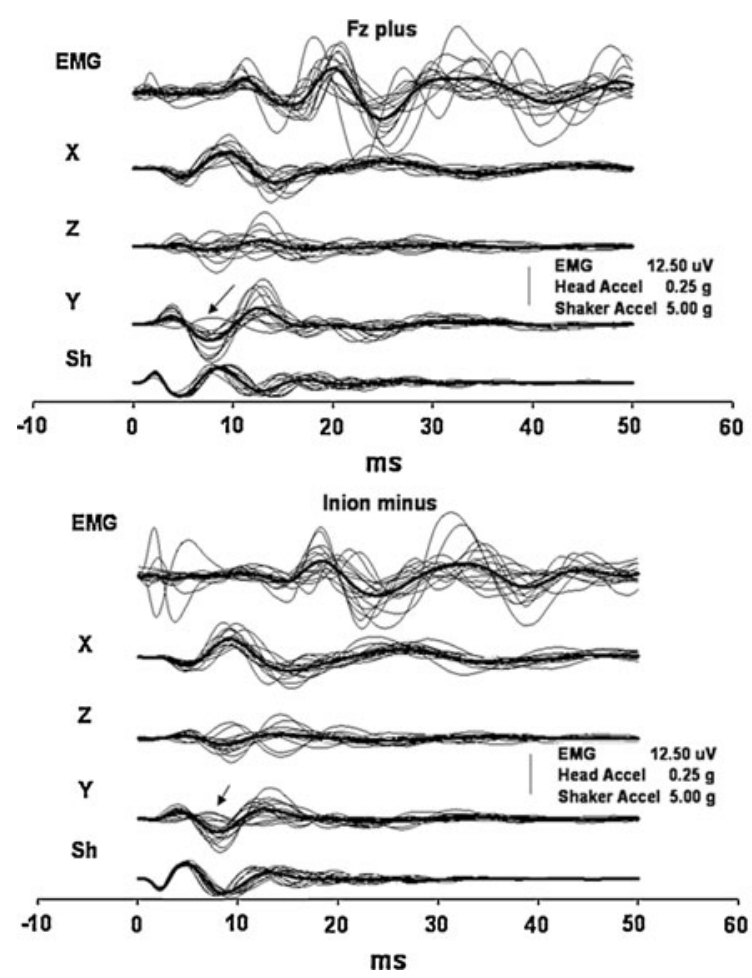

Fig. 1 EMG responses and accelerations of the head produced by stimuli directed backwards. The two panels at the top show the responses that were produced by positive impulses to the hairline at $\mathrm{Fz}$, and the panels at the bottom display the responses to negative impulses at the inion. The panels on the left side show the grand average (bold) traces and responses in the individual subjects superimposed. For better clarity, only the grand average traces are displayed in the panels on the right side. Please note that, unless otherwise specified, in each panel and in all figures the traces from top to bottom are arranged in the following order: EMG and head acceleration recorded at the left

coefficients were still moderately high $(+\mathrm{Fz} 0.65,+$ Inion 0.46, -Inion 0.46, -Fz 0.62).

On the other hand, the acceleration vectors along the head vertical $Z$ axis showed substantial variations between test subjects in all four experimental conditions, and the mean values of inter-individual correlation coefficients of $\mathrm{Z}$ acceleration traces during the same time interval were low or almost negligible $(+\mathrm{Fz} 0.05$, +Inion 0.23 , - Inion 0.36, $-\mathrm{Fz}$ 0.03). However, more detailed analysis revealed that from stimuli producing backward-directed head acceleration, i.e. $+\mathrm{Fz}$ and - Inion, the test subjects displayed two patterns of acceleration along the $Z$ axis and three patterns from stimuli producing forward-directed head acceleration, i.e. +Inion and -Fz. In general, more subjects showed an upward-directed acceleration vector for backward-directed stimuli and vice versa. Nevertheless, this entirely different out-of-phase behaviour of the acceleration vectors along the head $Z$ axis was not reflected in any parameters of the oVEMP responses as shown in Figs. 3 and 4.
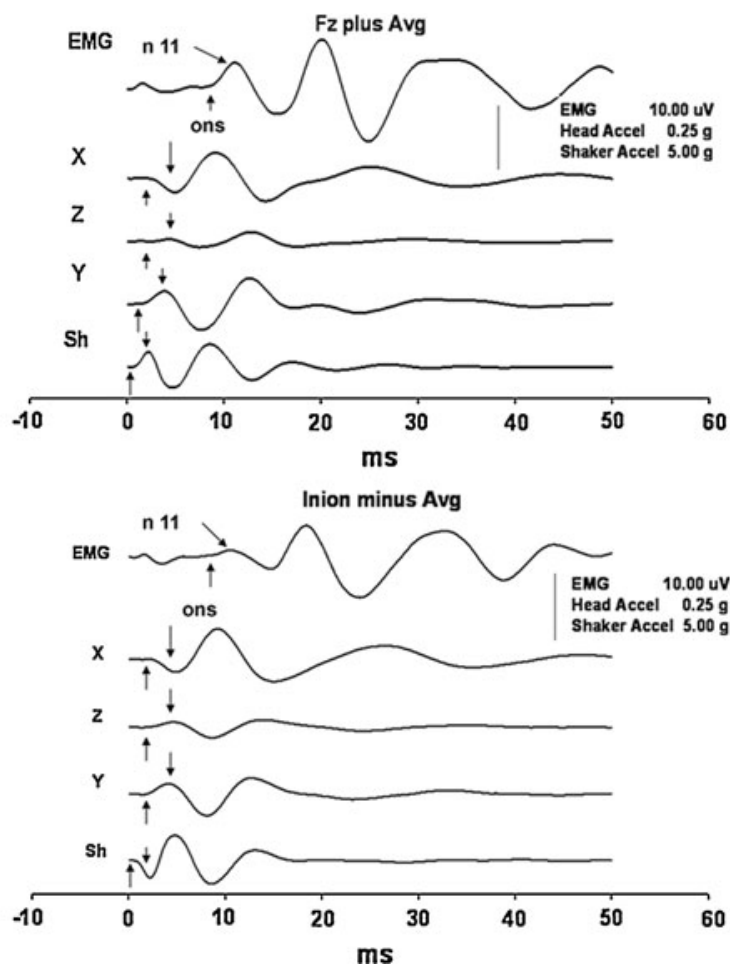

mastoid bone along its sagittal $(X)$, vertical $(Z)$ and transversal $(Y)$ axes; acceleration measured at the contact site of the stimulator. In all displays, upward deflection of the traces means a negative EMG signal; acceleration of the head forward for $(X)$, upward for $(Z)$, leftward for $(Y)$ axes and acceleration of the stimulator towards the head. The arrow in the panels on the left side, at $Y$ acceleration traces, marks the outlier cases. The arrows in the panels in the right side mark the acceleration onsets and the first acceleration peaks as well as the onsets and the first peaks of the oVEMPs

Accelerometry measurements at the mastoid. Comparison of the findings between individual ( $x, y$ and $z$ ) axes within the four experimental conditions

In all four experimental conditions, the latencies of the onsets and the first peaks were shortest for accelerations along the head $Y$ axis (Table 2). These differences between the $Y$ and $X$ axes were statistically significant in all four experimental conditions, both for the latencies' absolute values and for the values corrected to the mini-shaker acceleration onsets $(P<0.01)$. These differences were also significant between the $Y$ and $Z$ axes $(P<0.05)$ in all four experimental conditions, except for the onset by negative stimuli applied to the inion. Between the $X$ and $Z$ axes, there were no significant differences in the latencies except for the first peak produced by stimuli to $\mathrm{Fz}$ where $Z$ acceleration peaks occurred significantly earlier $(P<0.05)$. The acceleration magnitude at the first peak tended to be highest for the $\mathrm{X}$ axis and lowest for the $Z$ axis, 

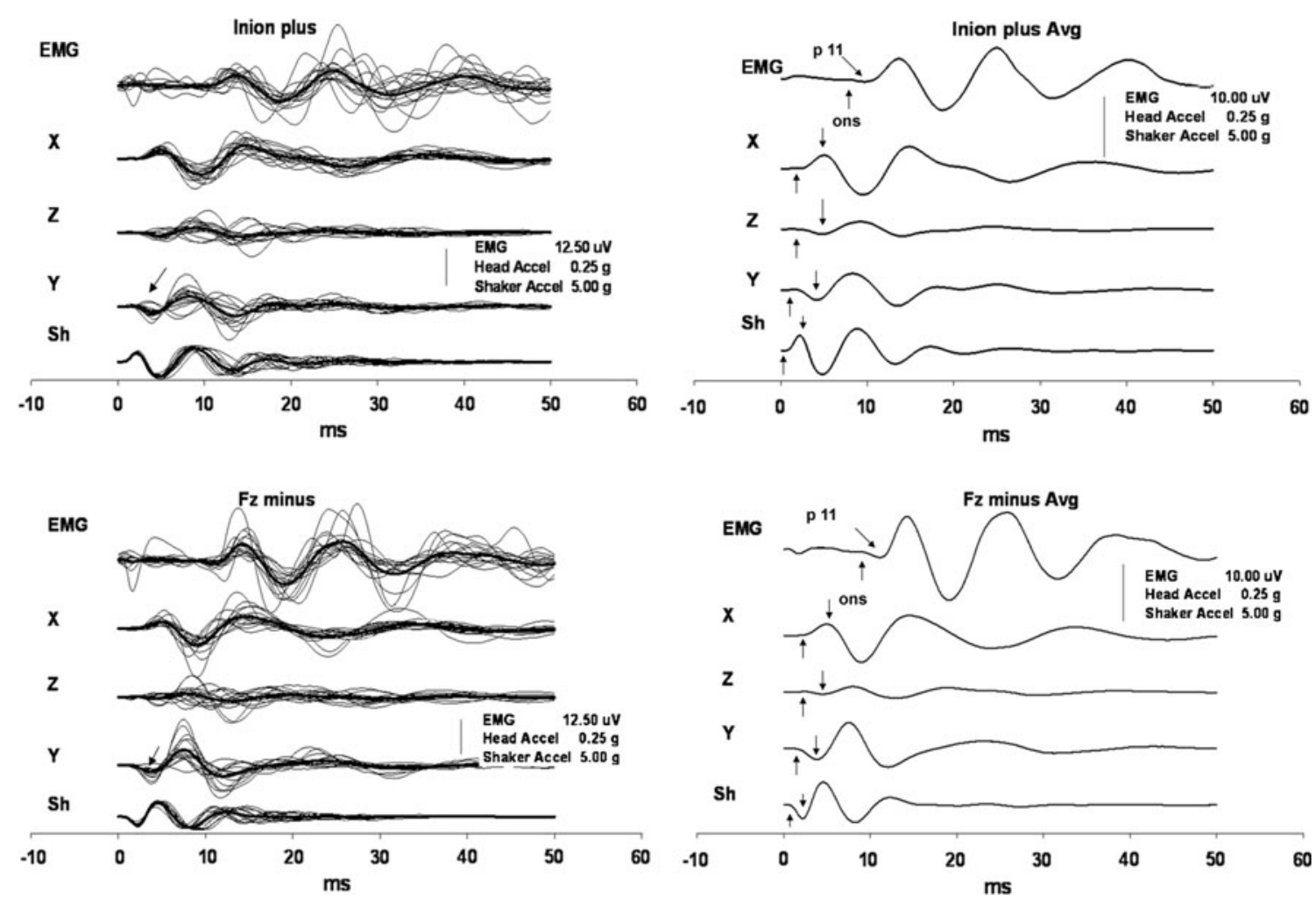

Fig. 2 EMG responses and accelerations of the head produced by stimuli directed forwards
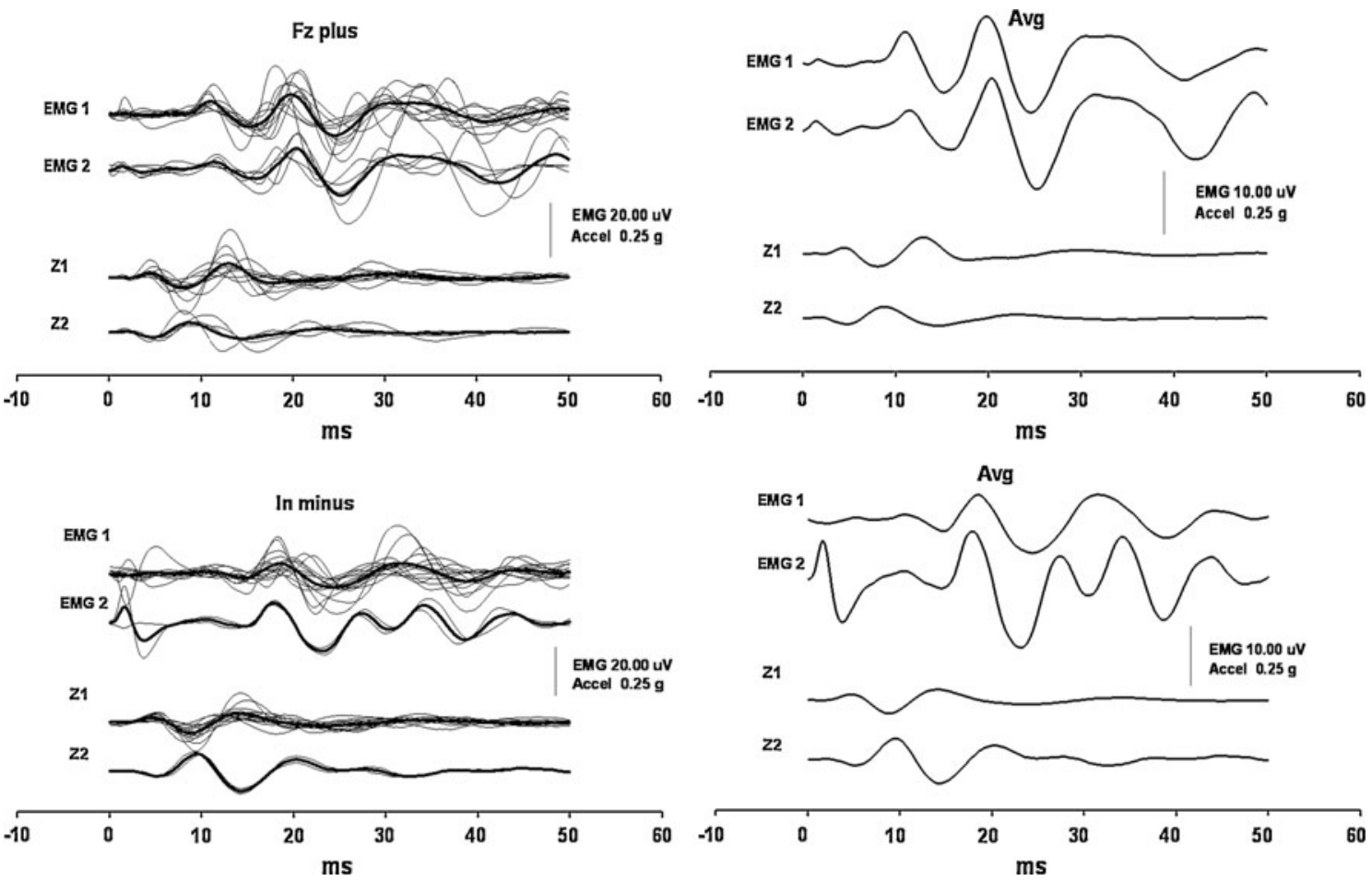

Fig. 3 Relationship of the EMG responses and accelerations of the head along the vertical axis produced by stimuli directed backwards. The two panels at the top show the responses produced by positive impulses at the hairline at Fz, and the panels at the bottom display the responses to negative impulses at the inion. The panels on the left side show the grand average (bold) traces and responses in the individual subjects superimposed. For better clarity, only the grand average traces are displayed in the panels

on the right side. In each panel, the upper two rows show the EMG responses and the bottom two the acceleration traces of the same subgroups. Both stimuli produced an initial upward-directed acceleration of the head in the majority of subjects. However, by positive stimuli to the hairline and by negative impulses to the inion, the acceleration of the head was opposite in five and two subjects, respectively. The oppositely directed vertical accelerations did not show any substantial influence on the EMG 

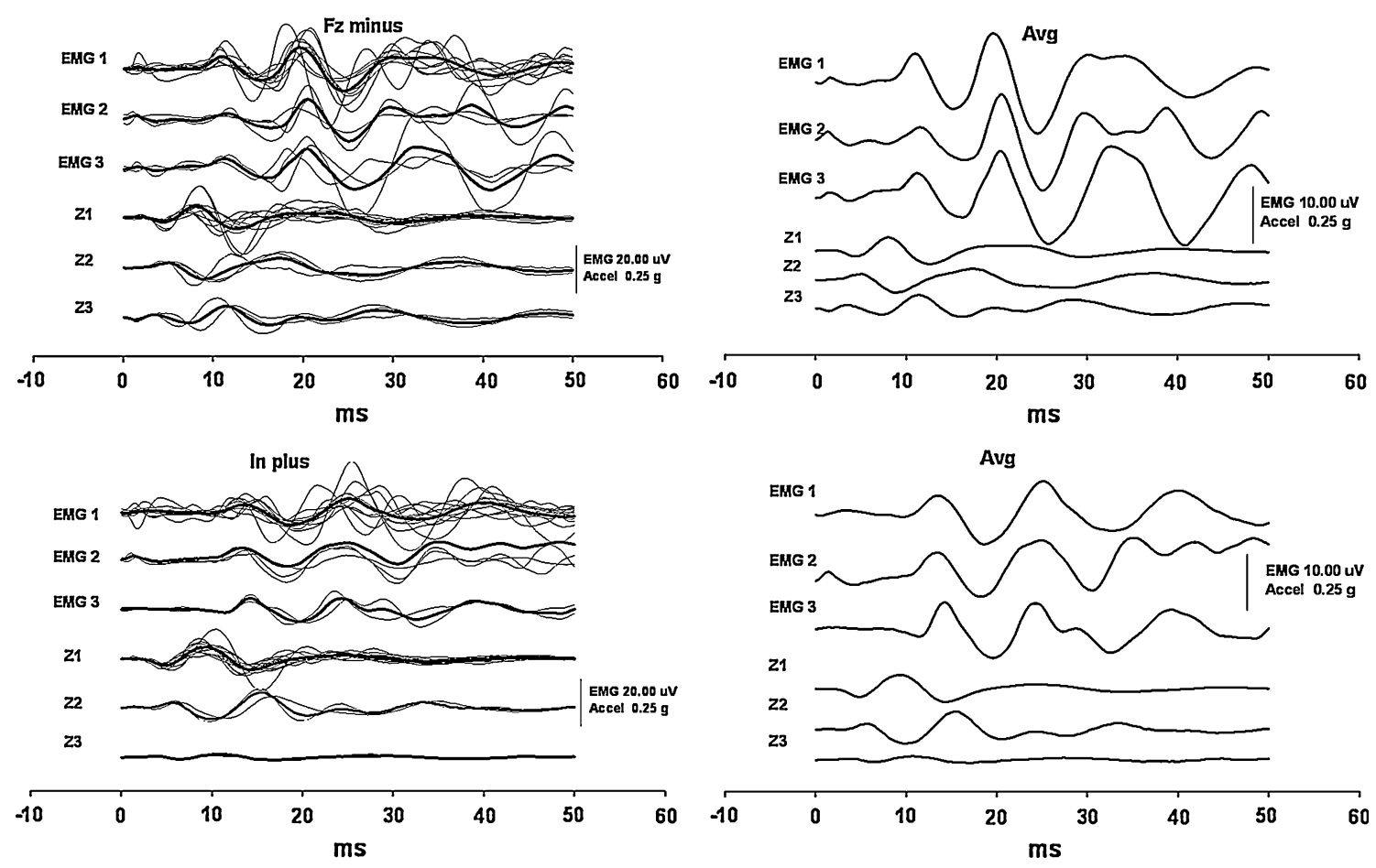

Fig. 4 Relationship of the EMG responses and accelerations of the head along the vertical axis produced by stimuli directed forwards. Two panels at the top show the responses produced by negative stimuli at the hairline at $\mathrm{Fz}$, and the panels at the bottom display the responses to positive impulses at the inion. Otherwise, the arrangement is the same as in Fig. 3. In each panel, the upper three rows show the EMG

and this difference was significant $(P<0.01)$. There were no significant differences between the $X$ and the $Y$ axes. Between the $Y$ and the $Z$ axis, the difference was significant only for positive impulses applied to the inion $(P<0.05)$.

\section{oVEMPs}

In general, there were no significant differences in the latencies and amplitudes between the oVEMP responses recorded from the left and right eye. Asymmetry ratios of peak-topeak amplitudes that were calculated using the standard Jongkees formula ${ }^{2}$ were well below 34 per cent. We therefore used the mean values from both eyes for subsequent analyses. However, there were a few exceptions when some subjects in one of the four conditions showed asymmetrical responses with low amplitude or distortion on one side. In such cases, the better response was used for analysis.

In all four conditions, the oVEMP responses showed consistent behaviour, and they copied the shapes of $X$ acceleration traces with a delay of about 5-6 ms, as shown in Figs. 1 and 2. The responses that were produced by backward-directed acceleration of the head showed an

\footnotetext{
$\overline{{ }^{2} \text { Asymmetry }}$ ratio [\%] $=[($ larger peak amplitude - smaller peak amplitude)/(larger peak amplitude + smaller peak amplitude $] \times 100$.
}

responses and the bottom three the acceleration traces of the same subgroups. Both stimuli produced an initial downward-directed acceleration of the head in the majority of subjects. However, two different patterns with initially upward-directed accelerations appeared in a subset of the subjects, without any influence on the EMG

Table 3 EMG latency findings

\begin{tabular}{|c|c|c|c|c|}
\hline & \multicolumn{2}{|c|}{ Acceleration backward } & \multicolumn{2}{|c|}{ Acceleration forward } \\
\hline & Fz plus & Inion minus & Inion plus & Fz minus \\
\hline \multicolumn{5}{|c|}{ Ons (ms) } \\
\hline Mean & 7.97 & 8.24 & 8.25 & 8.38 \\
\hline SD & 0.86 & 1.11 & 1.22 & 1.43 \\
\hline \multicolumn{5}{|c|}{$\mathrm{n} 11 / \mathrm{p} 11(\mathrm{~ms})$} \\
\hline Mean & 11.03 & 10.92 & 10.53 & 11.08 \\
\hline SD & 0.64 & 0.99 & 0.90 & 0.96 \\
\hline \multicolumn{5}{|c|}{$\mathrm{p} 14 / \mathrm{n} 14(\mathrm{~ms})$} \\
\hline Mean & 15.89 & 15.19 & $14.06^{*}$ & $14.24 *$ \\
\hline SD & 1.13 & 1.33 & 1.26 & 0.74 \\
\hline
\end{tabular}

ons onset, $n 11 / p 11$ first peaks of oVEMP responses produced by backward versus forward acceleration stimuli, $p 14 / n 14$ second peaks of oVEMP responses produced by backward versus forward acceleration stimuli

* Statistical significance level at $P<0.05$ for shorter latencies produced by forward-directed acceleration stimuli

initial negative peak at about $11 \mathrm{~ms}$. In contrast, the responses produced by forward-directed accelerations began with an initial positivity with approximately the same latency (Table 3). The subsequent peaks that occurred 


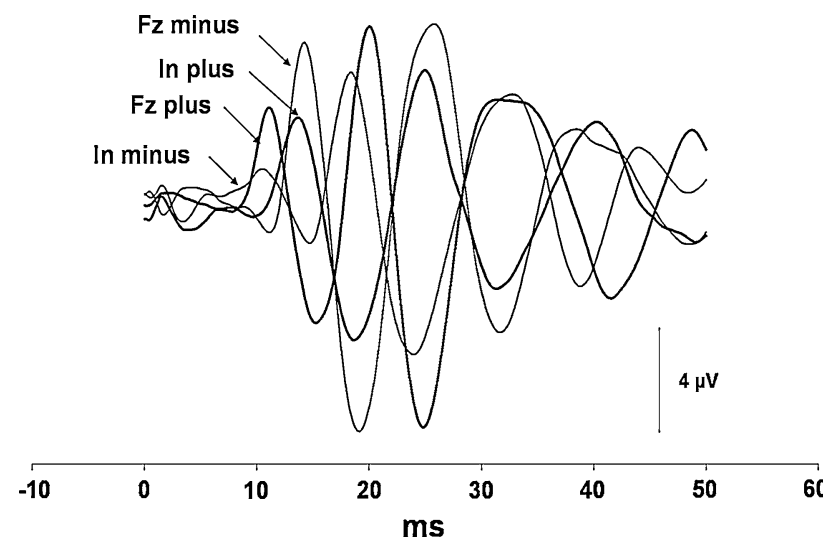

Fig. 5 Grand average EMG responses of 16 subjects in each of the four stimulation conditions. The bold traces show the responses produced by positive impulses, the responses produced by negative stimuli are displayed as thin traces. Please note that the responses produced by backward-directed head acceleration show the $\mathrm{n} 11$ peak, while those produced by forward-directed head acceleration have an oppositely directed $\mathrm{p} 11$ peak after their onset, and also the subsequent parts of the responses were almost completely out of phase

at about 14, 19, 24, 30 and $38 \mathrm{~ms}$ showed similar out-ofphase behaviour (Fig. 5). Only in two subjects, both women, were there some latency shifts in inion responses. They partially disrupted the otherwise perfect appearance of the out-of-phase behaviour of the responses that were still preserved in responses produced by stimuli with opposite polarity to Fz in these two subjects.

There were no significant differences either between latencies of the onsets or latencies of later-occurring subsequent peaks of the oVEMP responses in the four experimental conditions except for the second peak that occurred at about $14 \mathrm{~ms}$. Both the absolute latencies of this peak and its latency adjusted to the mini-shaker acceleration onset were significantly shorter for stimuli producing forward acceleration, i.e. positive impulses to the inion and negative impulses to $\mathrm{Fz}(P<0.05)$. However, there were no significant latency differences for this peak between conditions with the same acceleration direction as shown in Table 3.

The amplitude of the first oVEMP peak was significantly highest $(P<0.01)$ for positive stimuli to $\mathrm{Fz}$ in comparison with the other three experimental conditions, but there were no significant differences between the rest of the conditions. Amplitudes measured between the first and the second peak were higher for stimuli applied to Fz. These differences were significant $(P<0.01)$ except for + Inion versus $-\mathrm{Fz}$ as shown in Table 4.

Comparisons of the accelerometry and oVEMPs in head-free and head-fixed conditions

Accelerations of the head and the oVEMP responses tested in one of the subjects in all four experimental conditions
Table 4 oVEMP amplitude findings

\begin{tabular}{|c|c|c|c|c|}
\hline & \multicolumn{2}{|c|}{ Acceleration backward } & \multicolumn{2}{|c|}{ Acceleration forward } \\
\hline & Fz plus & Inion minus & Inion plus & Fz minus \\
\hline \multicolumn{5}{|c|}{ ons-n11/p11 ( $\mu \mathrm{V})$} \\
\hline Mean & $5.21 * *$ & 2.10 & 1.57 & 2.44 \\
\hline SD & 3.05 & 1.25 & 1.25 & 1.86 \\
\hline \multicolumn{5}{|c|}{ n11/p11-p14/n14 $(\mu \mathrm{V})]$} \\
\hline Mean & $10.85^{\# \#}$ & 4.70 & 6.08 & $9.00^{\#}$ \\
\hline SD & 4.92 & 2.33 & 2.38 & 5.36 \\
\hline
\end{tabular}

For abbreviations see Table 3

** Statistical significance level at $P<0.01$ for higher amplitudes of the first oVEMP peaks between $+\mathrm{Fz}$ and the rest of the conditions in the paired comparisons

\#\# Statistical significance level at $P<0.01$ for higher amplitudes of the second oVEMP peaks between $+\mathrm{Fz}$ versus +Inion and -Inion in the paired comparisons

\# Statistical significance level at $P<0.01$ for higher amplitudes of the second oVEMP peaks between $-\mathrm{Fz}$ versus - Inion in the paired comparisons

were almost entirely identical on visual assessment regardless of whether the head was free or fixed (Fig. 6).

Frequency analysis

Frequency analysis of the mean traces of the stimulus recorded at the contact site of the head and the mini-shaker and at the site of the mastoid, as well as the EMG (oVEMP) signal, showed that the power was approximately $100 \mathrm{~Hz}$ (the exact values were 117 for the mini-shaker; and $78 \mathrm{~Hz}$ for $X$ and $Z$ axes as well as EMG and $98 \mathrm{~Hz}$ for $Y$ axis) as shown in Fig. 7.

\section{Discussion}

In general, we found almost consistent behaviour in head acceleration in the horizontal plane and resultant oVEMP responses in the studied sample of normal subjects. The traces of the oVEMPs that were produced by oppositely directed head accelerations consistently showed the opposite phase.

\section{Accelerometry}

Regardless of the negative or the positive stimuli, the direction of the head acceleration always followed the direction of movement of the stimulation device. Owing to the spring-like mechanics of the neck, the tight contact caused by the pressure of the mini-shaker against the head resulted in the head and the device moving in synchrony (the elastic restoring forces of the skull might also play some role). The 


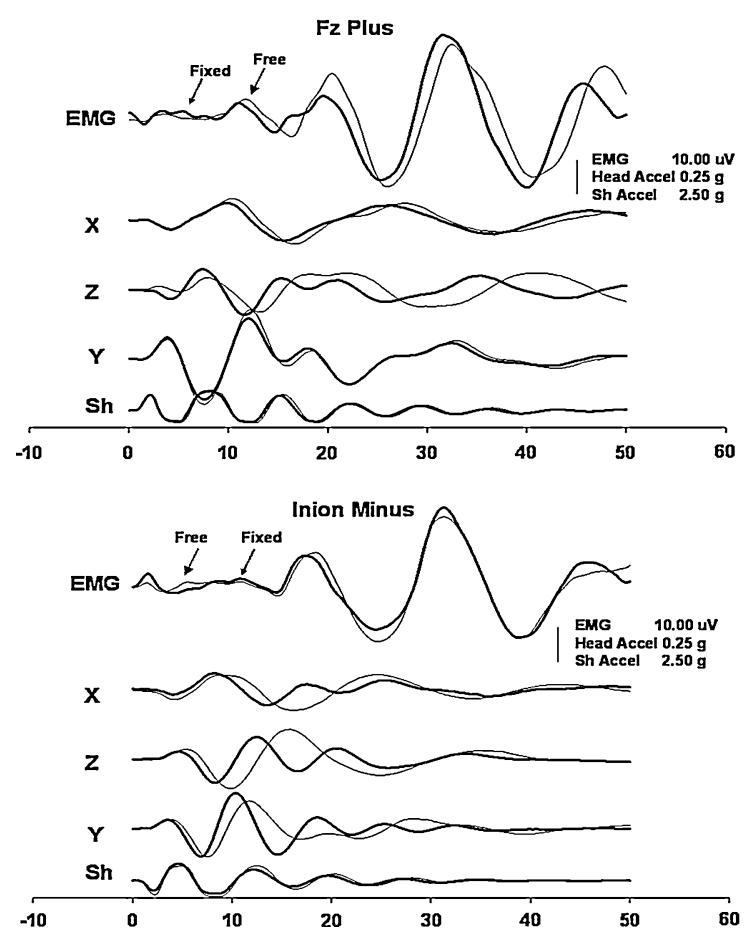

Fig. 6 EMG responses and accelerations of the head produced by head-free and head-fixed conditions in one of the subjects. Panels on the left and right sides show the responses produced by stimuli directed backwards and forwards, respectively. Thin traces display the respons-

acceleration traces on the mini-shaker and the head displayed a damped oscillation pattern.

The latency of mini-shaker acceleration onset was about $30 \mu$ s shorter for stimuli applied to the inion compared to stimulation at Fz. The reason for the earlier onset of the mini-shaker acceleration for stimuli applied to the inion might be the more compliant hair layer at this site in contrast to the less compliant bare skin at the hairline.

The magnitudes of the acceleration peaks recorded at the site of the mastoid were about two orders below those recorded at the contact site of the mini-shaker and the head. These values are similar to those described in previous studies by other authors (Iwasaki et al. 2008a; Todd et al. 2008a; Rosengren et al. 2009a, b).

The response of the skull to BC stimuli is complex. These mechanical stimuli can produce both elastic-compression waves and in-compressive vibration of the head. The displacements of the head can consist of three-dimensional translation and rotation. The size of these displacements would have been very small, at the order of about $10^{-6} \mathrm{~m}$, given the size and the duration of the accelerations which we have recorded on the heads (Todd et al. 2008a). At low frequencies below $1 \mathrm{kHz}$, the head and especially the skull base tends to move as a whole (Stenfelt and Håkansson 2000; Stenfelt and Goode 2005a, b). Nevertheless, the shell of the skull and its base tend to behave differ-
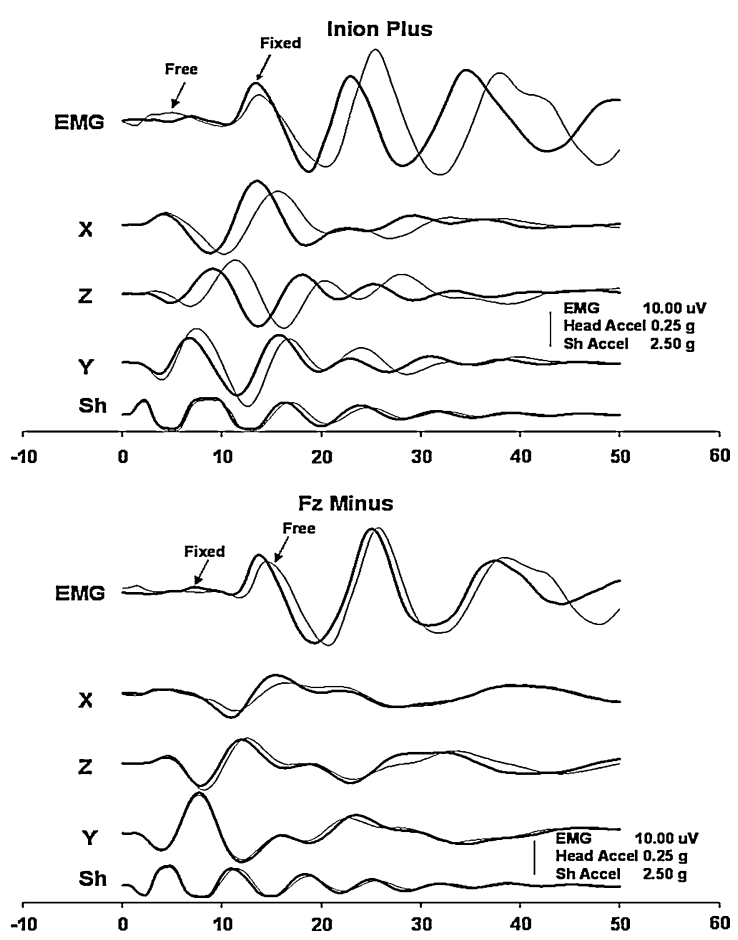

es produced in head-free conditions, and the bold traces show the responses produced during the experiment when the head was held fixed at the wall

ently, so the elastic-compressive waves in the shell can still be prominent even for low-frequency stimuli. However, the accelerometry findings in this study showed that, despite the low frequency of the stimulus at about $100 \mathrm{~Hz}$ and mode of its application, i.e. pressing the mini-shaker to the head, a significant portion of the elastic-compression waves was transmitted from the skull shell to the mastoid. We suspect that the reason for this is the site of the stimulation, i.e. the shell of the skull, because despite the highest magnitude of skull acceleration occurring along its $X$ axis, in contrast to studies that used $500-\mathrm{Hz}$ BC tone burst stimuli and head taps (Iwasaki et al. 2008a, 2009), the compression waves oriented along the head $Y$ and $Z$ axes were still present and showed significant magnitudes.

Interpretation of the accelerometric findings is not easy, but the following could be proposed: the skull is neither a homogeneous nor an isotropic mass body. Compliance of the skull bone can vary at different sites. Moreover, its anterior part, including the frontal, sphenoid, and the temporal bones are joined together by tightly ossified sutures. By contrast, the occipital bone at the base of the skull has a wedge shape. The lateral parts of this wedge are joined with the pyramidal parts of the temporal bones at least partly by softer fibrocartilaginous tissues.

Owing to the compliance of the skull shell bone, the applied force of stimulus first caused compressive-elastic 

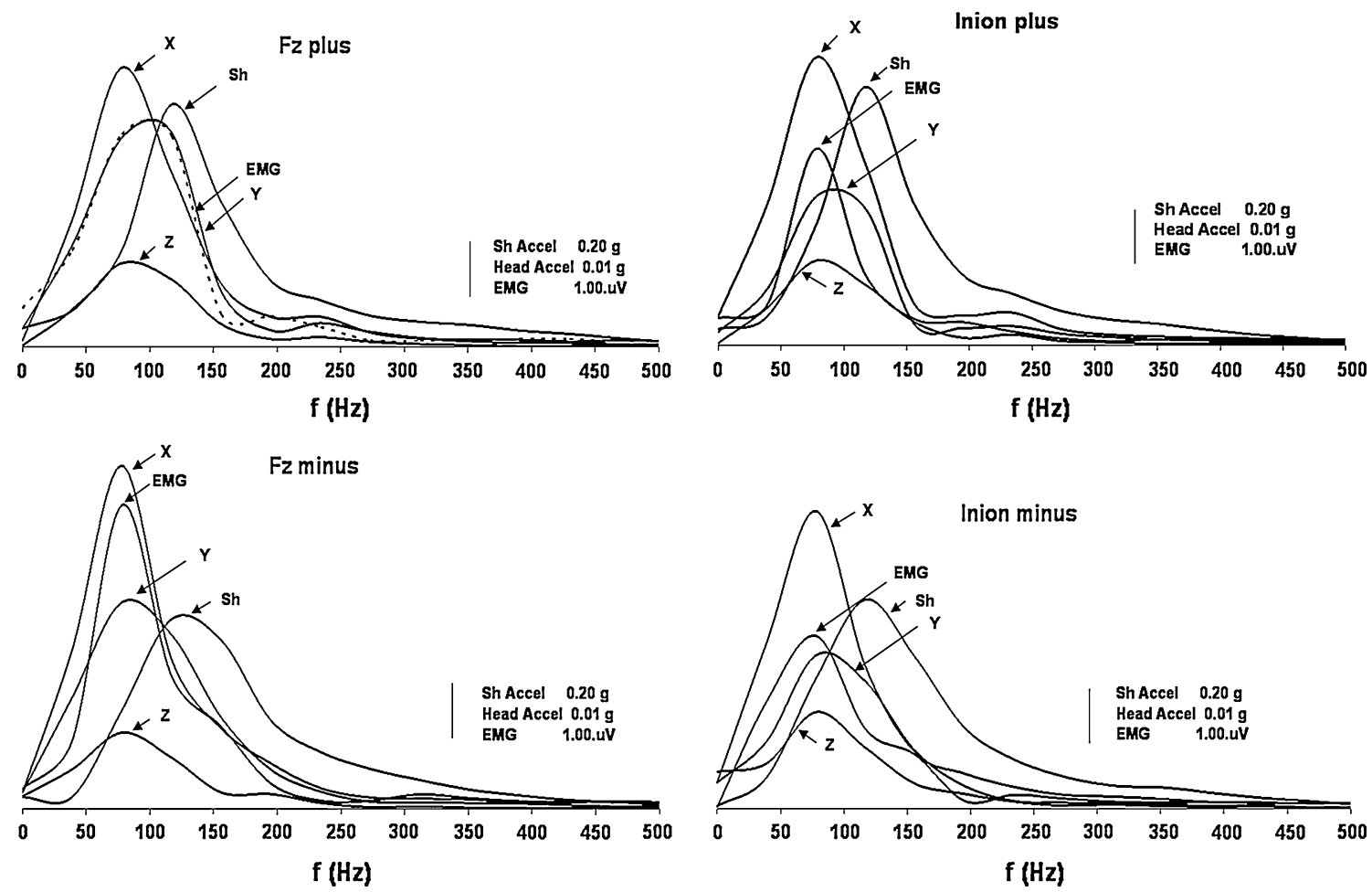

Fig. 7 Frequency analyses of stimuli and responses. Grand averages of the traces recorded by accelerometers and EMG signals are displayed here in their frequency domain. The two panels at the top show the stimuli and responses produced by positive impulses to the hairline

deformation of the skull, and only after exhaustion of this elastic freedom did acceleration in accordance with the direction of the stimulus follow. This could explain the longer latencies of the acceleration along the head $X$ axis in all four experimental conditions.

The reason for shorter latencies of acceleration along the $Y$ and $Z$ axes for stimulation at the $\mathrm{Fz}$ site compared to the inion might be the shorter transmission time for this elasticdeformation wave from the frontal shell to the mastoid bone through the hard dense bone structures in contrast to the softer connections in the path from the inion. Nevertheless, according to the latency differences found in this study, the differences in the conduction velocity could be only two- or several-fold faster, but should not reach values of the order suggested in one previous study (Lin et al. 2010).

The differences in the orientation of $\mathrm{Y}$ axis acceleration vectors produced by backward- and forward-directed stimuli suggest an entirely different mode of skull deformation by these oppositely directed stimuli. Owing to the tight osseous connections of the frontal and temporal bones, the initial elastic-deformation forces produced by stimuli applied to the hairline cause transient changes to the skull shape from spherical to ovoid. This leads to outwarddirected acceleration of the pyramids. On the other hand,

at Fz (left) and to the inion (right), and the panels at the bottom display the recordings produced by negative impulses at the corresponding sites

the forces acting at the inion might cause small displacements of the wedge-shaped basal part of the occipital bone with concomitant elongation and narrowing of fibrocartilaginous structures in the foramen lacerum and subsequent medially directed acceleration of the pyramids. We propose this model for the positive stimuli, and the opposite might happen with negative impulses.

Head acceleration direction in the utricular and saccular planes and the oVEMPs

The directions of the head accelerations along the anteroposterior and the transverse axes, i.e. in the main plane of the utricle, were with few exceptions the same in the tested subjects. On the other hand, there was a great variability in the acceleration along the head vertical axis, i.e. in the main saccular plane, recorded at the mastoid bone without any consistent trend between the subjects, the polarity of the stimulus, and the stimulation site. The oVEMP responses also showed consistent behaviour in their phases in relation to the head acceleration direction in the plane of the utricle, but not to the acceleration direction in the saccular plane. Thus, in general the head impulses that were initially directed backward (positive impulses to the hairline and negative impulses to the inion) also caused backward- and 
outward-directed acceleration at the mastoid bone. The resultant oVEMPs were consistently accompanied by an initially negative response with a mean latency of about $11 \mathrm{~ms}$, i.e. the $\mathrm{n} 11$ peak. Conversely, forward-directed accelerations of the head (negative impulses to the hairline and positive impulses to the inion) almost always caused inward-directed acceleration at the mastoid bone in addition to the forward-directed vector and produced an initially positive oVEMP response with approximately the same latency of about $11 \mathrm{~ms}$ but with opposite polarity, i.e. the p11 peak.

The EMG responses show initial negativity when recorded close to the motor point of the muscle and when the muscle is depolarized. Conversely, the inhibition of the ongoing muscle tonic activity is accompanied by positive potential (Colebatch and Rothwell 2004). Direct eye movement recordings have shown that the first oVEMP potential reflects the activity of the inferior oblique muscle (Welgampola et al. 2009). Thus, we might expect an excitatory response of the inferior oblique muscle to backwarddirected head acceleration (and vice versa).

The oVEMP potentials differed in their phases, i.e. polarities, but otherwise they were almost identical. Nevertheless, the magnitudes of the first two peaks were higher for stimulation at the $\mathrm{Fz}$ site. This might reflect better efficacy of stimulation at this site in comparison with the impulses applied to the inion. On the other hand, the lower amplitudes of the first positive inhibitory potentials produced by negative impulses to $\mathrm{Fz}$ and positive impulses to the inion might reflect the natural propensity of the vestibular system for enhancing the excitatory responses.

In addition, the latencies of the later peaks were almost identical regardless of the stimulus site or polarity. We suppose therefore that they reflect the activity of the same vestibular end-organ and the same pathways, i.e. the projections from the utricle.

On the other hand, a smaller proportion of the hair cells situated in the anterior part of the saccule have their polarization vectors oriented along the anteroposterior axis of the head. Thus, some small contribution to the responses from this part of the saccule cannot be excluded.

Due to the limitations of the acceleration recording mode, we cannot exclude the possibility that head taps caused some angular head displacement around the transverse axis. Such stimuli could activate the vertical canal afferents. The anterior and posterior canals and the utricle project strongly towards the oblique muscles, while the horizontal canals and saccule do not (Isu et al. 2000; Uchino et al. 1996). Nevertheless, if the oVEMPs in the current study were produced by stimulation of the vertical canals, the responses must have opposite polarity. Clearly, head rotation backward will activate the posterior canal VOR, which is associated with the inhibition of the inferior oblique muscle (and vice versa). Finally, the auxiliary experiment with the head-free versus head-fixed conditions in one subject did not show any important difference in head acceleration direction or oVEMP response phases. Although this experiment could not entirely eliminate head rotation, we think that this condition could at least reduce it considerably.

Mechanism by which BC stimuli activate vestibular receptors and the origin of oVEMP responses produced by these stimuli applied to the hairline and to the inion

Young et al. (1977) found that both canal and otolithic primary afferents can be activated by $\mathrm{BC}$ vibrations. Later, Curthoys et al. (2006) showed that these stimuli selectively activate irregular otolithic receptors. The precise mechanism by which $\mathrm{BC}$ sound activates the otololithic receptors is not clear. With regard to hearing of $\mathrm{BC}$ sound, some studies propose that the dominant mechanism in the initiation of the cochlear bone conduction response is the action of pressure waves in the cerebrospinal fluid. These waves would be transmitted via the physiological third windows to the inner ear fluids (Sohmer et al. 2000; Freeman et al. 2000; Sohmer and Freeman 2004). However, such a mechanism would preclude direction-dependent vestibular responses to $\mathrm{BC}$ vibration. Thus, it is most probable that the otololithic receptors are activated by virtue of their inertia. It has been shown that the resonance for the oVEMP response occurs with frequencies around $100 \mathrm{~Hz}$, where an extraordinary level of vestibular sensitivity to these responses has been found (Todd et al. 2008b). Both central and peripheral mechanisms might be responsible for this tuning. At the level of the sensory organs, the biomechanical properties of the saccular and utricular maculae are quite different. The ratio of saccular vs. utricular frequency resonance has been determined at 6:1 (Todd et al. 2009a). Owing to these peripheral biomechanical factors, the frequency tuning of the saccular afferents is close to $500 \mathrm{~Hz}$. Conversely, the best frequency for the utricle is around $100 \mathrm{~Hz}$ (Todd et al. 2009a). Finally, the central VOR pathways for the oVEMPs, the afferent neural system from the extra-ocular muscles play a role in the reinforcement of oVEMPs' tendency for resonance at $100 \mathrm{~Hz}$ (Todd et al. 2009b). The main frequency of the stimuli produced by our mini-shaker was about $100 \mathrm{~Hz}$, as was the skull vibration response that we recorded at the mastoid bone.

The direction of the applied acceleration along the head $X$ axis was the major determinant of the response. We conclude therefore that low-frequency vibrations of the head induced by impulses to the hairline and the inion probably activate utricular afferents with oppositely directed spatial polarization vectors. The resultant direction-dependent 
excitatory and inhibitory oVEMP responses most likely reflect activity of the utriculo-ocular VOR pathways.

Acknowledgments We thank Ian Curthoys, Michael Halmagyi, Leigh McGarvie, Ann Burgess, Yvette Smulders, Miriam Welgampola and Viera Saly for their intellectual and technical support.

Open Access This article is distributed under the terms of the Creative Commons Attribution Noncommercial License which permits any noncommercial use, distribution, and reproduction in any medium, provided the original author(s) and source are credited.

\section{References}

Angelaki DE, Dickman JD (2000) Spatiotemporal processing of linear acceleration: primary afferent and central vestibular neuron responses. J Neurophysiol 84:2113-2132

Aw ST, Todd MJ, Aw GE, Magnussen JS, Curthoys IS, Halmagyi GM (2006) Click-evoked vestibulo-ocular reflex: stimulus-response properties in superior canal dehiscence. Neurology 66:10791087. doi:01.wnl.0000204445.81884.c7

Baloh RW, Honrubia V (2001) Clinical neurophysiology of the vestibular system, 3rd edn. Oxford University Press, Oxford

Brantberg K, Tribukait A (2002) Vestibular-evoked myogenic potentials in response to laterally-directed skull taps. J Vestib Res 12:35-45

Brantberg K, Löfqvist L, Westin M, Tribukait A (2008) Skull tapinduced vestibular-evoked myogenic potentials: an ipsilateral vibration response and a bilateral head acceleration response? Clin Neurophysiol 119:2363-2369. doi:10.1016/j.clinph.2008. 02.026

Brantberg K, Westin M, Löfqvist L, Verrecchia L, Tribukait A (2009) Vestibular-evoked myogenic potentials in response to lateral skull taps are dependent on two different mechanisms. Clin Neurophysiol 120:974-979. doi:10.1016/j.clinph.2009.03.008

Cheng P-W, Chen C-C, Wang S-J, Young Y-H (2009) Acoustic, mechanical and galvanic modes elicit ocular vestibular-evoked myogenic potentials. Clin Neurophysiol 120:1841-1844. doi:10.1016/j.clinph.2009.08.002

Chihara Y, Iwasaki S, Ushio M, Murofushi T (2007) Vestibularevoked extraocular potentials by air-conducted sound: another clinical test for vestibular function. Clin Neurophysiol 118:27452751. doi:10.1016/j.clinph.2007.08.005

Colebatch JG, Rothwell JC (2004) Motor unit excitability changes mediating vestibulocollic reflexes in the sternocleidomastoid muscle. Clin Neurophysiol 115:2567-2573. doi:10.1016/j.clinph. 2004.06.012

Curthoys IS, Kim J, McPhedran SK, Camp AJ (2006) Bone-conducted vibration selectively activates irregular primary otolithic vestibular neurons in the guinea pig. Exp Brain Res 175:256-267. doi:10.1007/s00221-006-0544-1

Fernandez C, Goldberg JM (1976) Physiology of peripheral neurons innervating otolith organs of the squirrel monkey. II. Directional selectivity and force-response relations. J Neurophysiol 39:985-995

Freeman S, Sichel JY, Sohmer H (2000) Bone conduction experiments in animals-evidence for non-osseous mechanism. Hear Res 146:72-80

Goldberg JM, Fernandez C (1971) Physiology of peripheral neurons innervating semicircular canals of the squirrel monkey. I. Resting discharge and response to constant angular accelerations. J Neurophysiol 34:635-660

Halmagyi GM, McGarvie LA, Aw ST, Yavor RA, Todd MJ (2003) The click-evoked vestibulo-ocular reflex in superior semicircular canal dehiscence. Neurology 60:1172-1175
Highstein SM, Rabbitt RD, Holstein GR, Boyle RD (2005) Determinants of spatial and temporal coding by semicircular canal afferents. J Neurophysiol 93:2359-2370. doi:10.1152/jn.00533.2004

Isu N, Graf W, Sato H, Kushiro K, Zakir M, Imagawa M, Uchino Y (2000) Sacculo-ocular reflex connectivity in cats. Exp Brain Res 131:262-268

Iwasaki S, McGarvie LA, Halmagyi GM, Burgess AM, Kim J, Colebatch JG et al (2007) Head taps evoke a crossed vestibulo-ocular reflex. Neurology 68:1227-1229. doi:10.1212/01.wnl.0000259064. 80564.21

Iwasaki S, Smulders YE, Burgess AM, McGarvie LA, MacDougall HG, Halmagyi GM et al (2008a) Ocular vestibular-evoked myogenic potentials to bone-conducted vibration of the midline forehead at Fz in healthy subjects. Clin Neurophysiol 119:2135-2147. doi:10.1016/j.clinph.2008.05.028

Iwasaki S, Smulders YE, Burgess AM, McGarvie LA, MacDougall HG, Halmagyi GM et al (2008b) Ocular vestibular-evoked myogenic potentials in response to bone-conducted vibration of the midline forehead at Fz: a new indicator of unilateral otolithic loss. Audiol Neurotol 13(6):396-404

Iwasaki S, Chihara Y, Smulders YE, Burgess AM, Halmagyi GM, Curthoys IS et al (2009) The role of the superior vestibular nerve in generating ocular vestibular-evoked myogenic potentials to bone-conducted vibration at Fz. Clin Neurophysiol 120:588-593. doi:10.1016/j.clinph.2008.12.036

Jasper H (1958) Report of committee on reports of clinical exam in EEG. Electroencephalogr Clin Neurophysiol 10:370-375

Jombik P, Bahyl V (2005a) Short latency responses in the averaged electro-oculogram elicited by vibrational impulse stimuli applied to the skull: could they reflect vestibulo-ocular reflex function? J Neurol Neurosurg Psychiatry 76:222-228

Jombik P, Bahyl V (2005b) Short latency disconjugate vestibuloocular responses to transient stimuli in the audio frequency range. J Neurol Neurosurg Psychiatry 76:1398-1402

Jombik P, Bahyl V, Drobny M, Spodniak P (2008) Vestibulo-ocular (oVEMP) responses produced by bone-conducted sound stimuli applied to the mid-sagittal plane of the head. J Vestib Res 18:117128

Leigh RJ, Zee DS (2006) The neurology of eye movements, 4th edn. Oxford University Press, New York

Lin CM, Wang SJ, Young YH (2010) Ocular vestibular-evoked myogenic potentials via bone-conducted vibrations applied to various midsagittal cranial sites. Otol Neurotol 31(1):157-161

Manzari L, Tedesco AR, Burgess AM, Curthoys IS (2010) Ocular and cervical vestibular-evoked myogenic potentials to boneconducted vibration in Ménière's disease during quiescence vs. during acute attacks. Clin Neurophysiol 121(7):1092-1101. doi:10.1016/j.clinph.2010.02.003

Raphan T, Cohen B (2002) The vestibulo-ocular reflex in three dimensions. Exp Brain Res 45:1-27

Rosengren SM, Todd NPM, Colebatch JG (2005) Vestibular-evoked extraocular potentials produced by stimulation with bone-conducted sound. Clin Neurophysiol 116:1938-1948. doi:10.1016/ j.clinph.2005.03.019

Rosengren SM, Nogajski JH, Cremer PD, Colebatch JG (2007) Delayed vestibular-evoked responses to the eyes and neck in a patient with an isolated brainstem lesion. Clin Neurophysiol 118(9):2112-2116. doi:10.1016/j.clinph.2007.05.068

Rosengren SM, Aw ST, Halmagyi GM, Todd NPM, Colebatch JG (2008) Ocular vestibular-evoked myogenic potentials (oVEMPs) in superior canal dehiscence. J Neurol Neurosurg Psychiatry 79(5):559-566

Rosengren SM, Jombik P, Halmagyi GM, Colebatch JG (2009a) Galvanic ocular vestibular-evoked myogenic potentials provide new insight into vestibulo-ocular reflexes and unilateral vestibular loss. Clin Neurophysiol 120:569-580. doi:10.1016/j.clinph.2008.12.001 
Rosengren SM, Todd NPM, Colebatch JG (2009b) Vestibular-evoked myogenic potentials evoked by brief interaural head acceleration: properties and possible origin. J Appl Physiol 107:841-852

Sohmer H, Freeman S (2004) Further evidence for a fluid pathway during bone conduction auditory stimulation. Hear Res 193:105-110

Sohmer H, Freeman S, Geal-Dor M, Adelman C, Savion I (2000) Bone conduction experiments in humans-a fluid pathway from bone to ear. Hear Res 146:81-88

Stenfelt S, Goode RL (2005a) Transmission properties of bone-conducted sound: measurements in cadaver heads. J Acoust Soc Am 118(4):2373-2391

Stenfelt S, Goode RL (2005b) Bone-conducted sound: physiological and clinical aspects. Otol Neurotol 26:1245-1261

Stenfelt S, Håkansson B (2000) Vibration characteristics of bone-conducted sound in vitro. J Acoust Soc Am 107:422-431

Todd NPM, Rosengren SM, Aw ST, Colebatch JG (2007) Ocular vestibular-evoked myogenic potentials (oVEMPs) produced by airand bone-conducted sound. Clin Neurophysiol 118:381-390. doi:10.1016/j.clinph.2006.09.025

Todd NPM, Rosengren SM, Colebatch JG (2008a) Ocular vestibularevoked myogenic potentials (oVEMPs) produced by impulsive transmastoid accelerations. Clin Neurophysiol 119:1638-1651. doi:10.1016/j.clinph.2008.03.009

Todd NPM, Rosengren SM, Colebatch JG (2008b) Tuning and sensitivity of the human vestibular system to low-frequency vibration. Neurosci Lett 444(1):36-41
Todd NPM, Rosengren SM, Colebatch JG (2009a) A utricular origin of frequency tuning to low-frequency vibration in the human vestibular system? Neurosci Lett 451(3):175-180

Todd NPM, Rosengren SM, Govender S, Colebatch JG (2009b) Lowfrequency tuning in the human vestibular-ocular projection is determined by both peripheral and central mechanisms. Neurosci Lett 458:43-47

Uchino Y, Sasaki M, Sato H, Imagawa M, Suwa H, Isu N (1996) Utriculo-ocular reflex arc of the cat. J Neurophysiol 76:1896-1903

Wang S-J, Weng W-J, Jaw F-S, Young Y-H (2010) Ocular and cervical vestibular-evoked myogenic potentials: a study to determine whether air- or bone-conducted stimuli are optimal. Ear Hearing 31(2):283-288. doi:10.1097/AUD.0b013e3181bdbac0

Welgampola MS (2008) Evoked potential testing in neuro-otology. Curr Opin Neurol 21(1):29-35

Welgampola MS, Myrie OA, Minor LB, Carey JP (2008) Vestibularevoked myogenic potential thresholds normalize on plugging superior canal dehiscence. Neurology 70(6):464-472. doi:10.1016/j.clinph.2008.06.020

Welgampola MS, Migliaccio AA, Myrie OA, Minor LB, Carey JP (2009) The human sound-evoked vestibulo-ocular reflex and its electromyographic correlate. Clin Neurophysiol 120:158-166. doi:10.1016/j.clinph.2008.06.020

Young ED, Fernández C, Goldberg JM (1977) Responses of the squirrel monkey vestibular neurons to audio-frequency sound and head vibration. Acta Otolaryngol (Stockh) 84:352-360 